\title{
An improved multi-value cellular automata model for mixed bicycle traffic flow on campus
}

\author{
Bi-Xiang Li ${ }^{\dagger}, 1, \mathrm{a}$ and Lan Fang ${ }^{2, \mathrm{~b}}$ \\ ${ }^{1}$ Department of Information Engineering, \\ City College of Wuhan University of Science and Technology, \\ Wuhan 430083, China \\ ${ }^{2}$ Department of Humanity, \\ City College of Wuhan University of Science and Technology, \\ Wuhan 430083, China \\ †e-mail:lbx153@163.com \\ be-mail:79610336@qq.com
}

\begin{abstract}
Firstly, this paper analyzes the defaults of traffic model applying in campus road in China, such as lacking simulation of vehicles, pedestrians and bicycles under mixed traffic flow. Secondly, the paper proposes an improved multi-value cellular automata model for revealing complex traffic phenomena of pedestrian-bicycle, pedestrian-vehicle and bicycle-vehicle mixed traffic flow. The model includes different forward rules and drift rules for different traffic types under campus roads. Thirdly, considering the mutual interference among different traffic types, it is named different interaction behavior. Finally, the difference of the degree of interaction interference among different traffic types is simulated and analyzed. Through qualitative analysis on traffic fundamental diagram, the complex mixed traffic flow phenomena is simulated by this model successfully, such as overtaking, drift, interaction, free flow, synchronized flow, congestion flow, slow traffic on the right side, etc. Therefore, this research is not only have a profound theoretical and practical value for campus security, but also have the function of reference and guidance for urban mixed traffic network.
\end{abstract}

Keywords: Cellular automata model; Multi-value; Mixed traffic; Bicycle.

\section{Introduction}

Mixed traffic is very popular on campus road in China, which mainly includes pedestrians, bicycles, e-bicycles, vehicles, etc. These road users all share the campus road, haven't the specific vehicle lane, especially the time of class time, the mix traffic flow is much larger than the other time, mixed traffic is influenced with each other, it will result in interference and conflict easily, even will result in traffic congestion and accident. How to relieve these congestion and accident, it is improved by intelligent transportation system, which reappear the complex traffic phenomenon, computer is a tool for simulating these micro traffic phenomenon, researches proposed cellular automaton----NS model [1], the micro 
traffic model set pedestrian or vehicle as a uniform unit, which could describe the unit's micro behavior, so revealing the complex ruler of mixed traffic flow, helping traffic designers and administrators for improving traffic design, planning and evacuation. Nowadays, these micro simulations model have been applying in highways, metro, urban roads, for researching traffic behavior model, for improving traffic congestion and traffic accident. But few researchers focus on mixed traffic problems on campus road.

NS model, which reproduced a simple ruler for describe the traffic phenomena between vehicles in highway, so this model only simulated simple vehicle flow on highway road. Multi-value cellular automata model was proposed by Noshinari [2], which was named BCA model. The road was divided with several cells by this model, every cells could occupy $M$ vehicles, so the model was required as $M$ lanes of road model, which couldn't define the road change ruler. The highest speed is 1 in the BCA model. Noshinari and Takahashi $[3,4]$ extend the BCA model, the highest speed was 2, which was named EBCA model. The EBCA model included EBCA1 model and EBCA2 model, the vehicle's speed was 1 owed priority in EBCA1 model, the vehicle's speed was 2 owed priorities in EBCA2 model. Rui JIANG [5] proposed stochastic randomization, and used to model bicycle flow in EBCA model, which simulated the bicycle flow successfully. Bin JIA[6] used EBCA1 and EBCA2 model to simulate bicycle flow, acquired the bicycle's fundamental diagram and spacetime plots under different bicycle flow density, the result showed the simulation was accord with the observed result on urban road.

The above research fruit promote the development of ITS, and suitable for some urban roads, the research point was centering on vehicles, but obliviously exists much defaults on campus road, which road users are mainly pedestrians and bicycles, these mature traffic software couldn't simulate the complex traffic phenomena on camps. This paper is based on NS model, is proposed an improved multi-value CA model under the behavior of mixed bicycle traffic flow on campus, which could simulate the reaction between vehicle flow, pedestrian flow and bicycle flow under large scale, and irregularity behavior of vehicles, pedestrians and bicycles influence on campus road.

\section{Models}

\subsection{Definition of cell size and dimension}

The campus road is much different from urban road, which mainly includes pedestrians, bicycles, cars etc. These different traffic type's size and speed is much different, for describing these traffic type accurately, every cell is $0.5 \mathrm{~m}$ 
length and $0.5 \mathrm{~m}$ width, every car occupies $7 \mathrm{~m}$ length, $2.5 \mathrm{~m}$ width, every bicycle occupies $2 \mathrm{~m}$ length, $1 \mathrm{~m}$ width, very pedestrian occupies $1 \mathrm{~m}$ length, $1 \mathrm{~m}$ width.

\subsection{Updating rules for pedestrians, bicycles and cars}

The pedestrian is the most complex behavior rule in all road users, which has group behavior, randomness, etc. Contrast to the other traffic type, the forward rule is much different from the other road users [7].

The pedestrians forward rules:

Setp1: Acceleration:

if $d_{n}^{\text {eff }}>v_{n}$ then $v_{n}=\min \left(v_{n}+a_{p}, v_{\text {max }}\right)$.

$\mathrm{d}_{\mathrm{n}}^{\text {eff }}=\mathrm{x}_{\mathrm{l}, \mathrm{n}}-\mathrm{x}_{\mathrm{n}}-\mathrm{d}_{\mathrm{l}}$, which denotes the effective space gap between two traffic objects. $a_{p}$ represents the acceleration of the pedestrian. $p$ represents the randomization probability of the pedestrian.

Setp2: Deceleration:

if $d_{n}^{\text {eff }} \leq v_{n}$ then $v_{n}=d_{n}^{\text {eff }}$.

Setp3: Randomization:

if $\left(\operatorname{rand}(\quad)<\mathrm{p}\right.$ then $\mathrm{v}_{\mathrm{n}}=\max \left(\mathrm{v}_{\mathrm{n}}-1,0\right)$.

Setp4: Update position:

$\mathrm{x}_{\mathrm{n}}(\mathrm{t}+1)=\mathrm{x}_{\mathrm{n}}(\mathrm{t})+\mathrm{v}_{\mathrm{n}}$.

The bicycle's behavior is similar with pedestrians, but it has uniform behavior, such as s-shaped trajectory, unsteadiness, and so on [8,9].

The bicycles forward movement rules:

$\mathrm{L} \rightarrow \mathrm{R}:$ if $\mathrm{d}_{\mathrm{n}}^{\text {right }}>\min \left(\mathrm{d}_{\mathrm{n}}^{\text {eff }}, \mathrm{v}_{\mathrm{n}}\right)$ and $\mathrm{d}_{\mathrm{n}}^{\text {right }}>\mathrm{v}_{\mathrm{n}}^{\text {bright }}$,

$\mathrm{R} \rightarrow \mathrm{L}:$ if $\mathrm{d}_{\mathrm{n}}^{\text {right }}>\mathrm{v}_{\mathrm{n}}^{\text {front }}$ and $\mathrm{v}_{\mathrm{n}}>\min \left(\mathrm{d}_{\mathrm{n}}^{\text {eff }}, \mathrm{v}_{\mathrm{n}}\right)$ and $\mathrm{d}_{\mathrm{n}}^{\text {bleft }}>\mathrm{v}_{\mathrm{n}}^{\text {bleft }}$

$\mathrm{L} \rightarrow \mathrm{R}$ denotes bicycles turn left to right movement, $\mathrm{R} \rightarrow \mathrm{L}$ denotes bicycles turn right to left movement, and bleft represents on the left hand and back hand.

Through the improved multi-value automata model establishment, the bicycles' acceleration ruler, deceleration ruler, randomization ruler and update forward position ruler as follows:

Setp1: Acceleration:

$\mathrm{v}_{\mathrm{n}}(\mathrm{t}+1)=\min \left(\mathrm{v}_{\mathrm{n}}(\mathrm{t})+\mathrm{a}, \mathrm{v}_{\max }\right)$.

Setp2: Deceleration:

$\mathrm{v}_{\mathrm{n}}(\mathrm{t}+1)=\min \left(\mathrm{d}_{\mathrm{n}}^{\text {eff }}(\mathrm{t})+\mathrm{v}_{\mathrm{n}}(\mathrm{t}+1)\right)$

Setp3: Randomization:

If $($ rand ()$\leq \mathrm{p})$ then $\mathrm{v}_{\mathrm{n}}(\mathrm{t}+1)=\max \left(0, \mathrm{v}_{\mathrm{n}}(\mathrm{t}+1)-1\right)$.

Setp4: Update forward position:

$\mathrm{x}_{\mathrm{n}}(\mathrm{t}+1)=\mathrm{v}_{\mathrm{n}}(\mathrm{t})+\mathrm{v}_{\mathrm{n}}(\mathrm{t}+1)$.

As the above rulers, a presents the acceleration of bicycle,p presents the randomization probability of the bicycle.

The car's forward rule, acceleration rule and the other updating rules are accord with Liu M proposed vehicle rules [10]. 


\section{The video-survey and simulation results}

Nanhu campus is one of campus in Wuhan University of Technology, which has the most pedestrians and bicycles. The intersection between new 1 and new 2 teaching building is the most pedestrians and bicycles, especially in time of class and after time of class. The cars, pedestrians and bicycles data were collected by video graphic survey on Nanhu campus in March, 2014 [11,12]. The vertical direction and horizontal direction both included 13 mark lines, the intersection's length is $14 \mathrm{~m}$, width is $13 \mathrm{~m}$, and the area is $182 \mathrm{~m}^{2}$.

\subsection{The video-survey results}

\subsubsection{Car's flow and speed}

Through the video software statistics analyzes, between 8:00 and 9:30, and from 10:00 to $11: 30$, the observed car number is only 5 per min and 3 per min respectively. And the speed of car is much slower on campus than that of other urban roads, the average speed range from $4 \mathrm{~m} / \mathrm{s}$ to $4.48 \mathrm{~m} / \mathrm{s}$ on campus, the average speed range usually from $6 \mathrm{~m} / \mathrm{s}$ to $16.6 \mathrm{~m} / \mathrm{s}$ on urban roads.

\subsubsection{Pedestrian's flow and speed}

Between 7:30-12:00, the max is 97 per min, the average is 10.92 per min, the total number is 2948. The number of pedestrian's flow is relative with school schedule. From 7:30 to 8:00, the pedestrian flow is the highest, the total flow is 1110 pedestrians, the road users are not only include students and teachers, but also include the other persons whose work in around the campus and doing morning exercise persons, etc. From 9:30 to 10:00, the pedestrian flow is the second highest, the total is 506 pedestrians, the flow is mainly include students, whose ending the second class and starting the third class. From 11:30 to 12:00, the pedestrian flow is the third highest, the total is 330 pedestrians.

As shown in the Fig 1, the pedestrian speed frequency distribution histogram is drawn by SPSS software: 


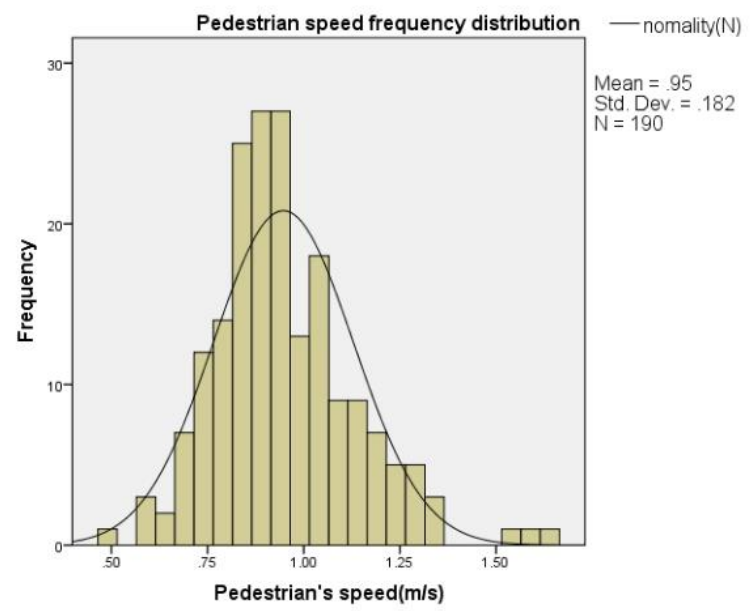

Fig 1: Frequency Distribution of Observed through Pedestrian speeds

The SPSS'S histogram and descriptive were used to test the normality of pedestrian speed data. The results indicated that skewness $=0.797$, Kurtosis $=1.550$, so the pedestrian speed obeyed normal distribution at the $5 \%$ significance level. So the campus intersection is much different from city transportation. In normal transportation condition, the pedestrian's speed isn't change at all. Based on the statistic analyzing, the highest speed of pedestrian is mainly in 7:30-8:00, because some students get up after 7:30, and have breakfast before 8:00, so they are very urgent in the first class, some of them even running to class.

\subsubsection{Bicycle's flow and speed}

Bicycle is a flexible and practical transportation tool, fitting for short distance trip, which is very common is campus roads, especially in large area universities, dorm, teaching building are usually far from with each other, so many students choose bicycle to go to class.

Based on statistics analyzing, between 7:30-12:00,the max is 107 per min, the average was 10.65 per min, the total number was 2875 .So as the same of pedestrian flow, as shown in the figure 5,the bicycle flow peaks was three time periods, from 7:30 to 8:00, 9:30 to 10:00 and 11:30 to 12:00.

Contrast to vehicle, bicycle is randomness, unsteadiness swing, slow speed. Contrast to pedestrian, bicycle's speed are usually higher, bicycle's behavior is much same as pedestrian. Through the statistics analyzing, as shown in the Fig 2, the results indicated that skewness $=0.362$, kurtosis $=0.367$, so the bicycle's (or the cyclist's) speed obeyed normal distribution at the 5\% significance level. 


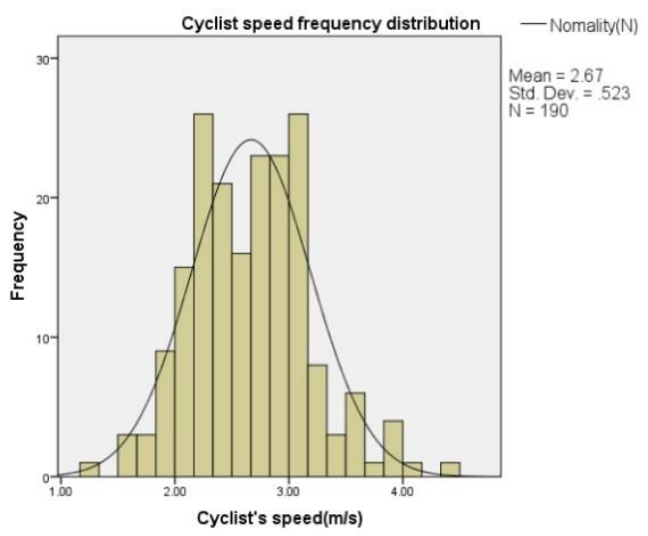

Fig 2: Frequency Distribution of Observed through Bicycle speeds

\subsection{The comparison between video-survey and simulation results}

For verifying the model's suitability and effectiveness, this paper selected 30 min video was selected as the experimental data, which was between 8:00 and 9:30 in east gate in Nanhu campus of Wuhan University of Technology.

The speed of three traffic objects were plotted under different distributions, as shown in Fig 3, which shows that the average speed of observed video are more disperse distribution than simulation result under the improved multi-value cellular automata model, but the comparison is a little different, so the speed distribution of cars, pedestrians and bicycles of simulation is consistent with observed results. These findings illustrate that this introduced model could reappear the actual speed of bicycle mixed traffic flow.

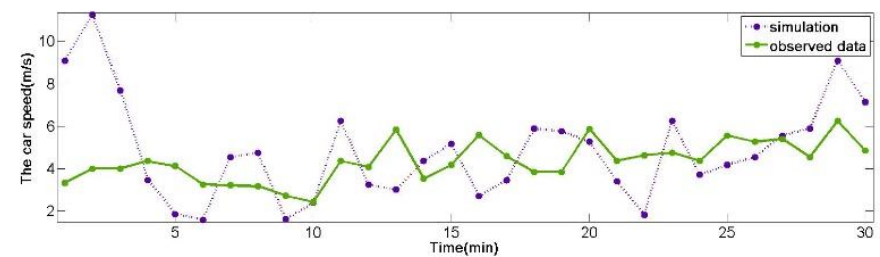

(a) car

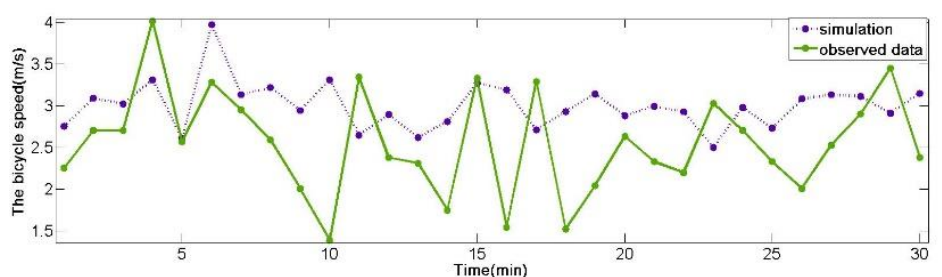

(b) bicycle 


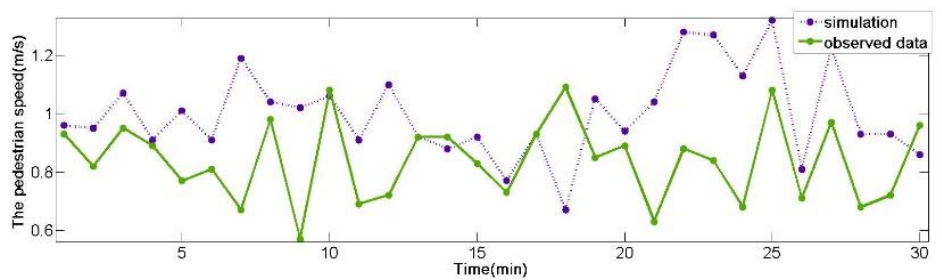

(c) pedestrian

Fig.3: Average Speed for Simulation Data and Observed Data

\section{Conclusions}

In summary, the improved multi-value cellular automata model simulates the complex mixed traffic flow on campus in china, the experiment result conforms the model reappear the campus traffic successfully, which helping mixed traffic's evacuation, relieving the traffic congestion and accident on campus road. The future work will design much more fined cell, considering the pedestrian and bicycle's complex behavior in different roads and visions.

\section{Acknowledgment}

This research was supported in part by the science and technology research project of Education Department of Hubei Providence, China (B2015359, B2015360).

\section{References}

[1] K. Nagel, M. Schreckenberg, A cellular automaton model for freeway traffic, J. Phys. I France 2 (12) (1992) 2221-2229.

[2] Nishinari K, Takahashi D. Analytical properties of ultradiscrete Burgers equation and rule-184 cellular automaton[J]. Journal of Physics A: Mathematical and General, 1998, 31(24): 5439.

[3] Nishinari K, Takahashi D. A new deterministic CA model for traffic flow with multiple states[J]. Journal of Physics A: Mathematical and general, 1999, 32(1): 93.

[4] Nishinari K, Takahashi D. Multi-value cellular automaton models and metastable states in a congested phase[J]. Journal of Physics A: Mathematical and general, 2000, 33(43): 7709.

[5] Jiang R, Jia B, Wu Q S. Stochastic multi-value cellular automata models for bicycle flow[J]. Journal of Physics A: Mathematical and General, 2004, 37(6): 2063 
[6] Jia B, Li X G, Jiang R, et al. Multi-value cellular automata model for mixed bicycle flow[J]. The European Physical Journal B-Condensed Matter and Complex Systems, 2007, 56(3): 247-252.

[5] Meng J, Dai S, Dong L, et al. Cellular automaton model for mixed traffic flow with motorcycles[J]. Physica A: Statistical Mechanics and its Applications, 2007, 380: 470-480.

[6] L.W. Lan, C.W. Chang, Inhomogeneous cellular automata modeling for mixed traffic with cars and motorcycles, J. Adv. Transp. 39 (3) (2005) 323349.

[7] Luo Z, Lin Y, Guo C. Operational characteristics of mixed traffic flow under bi-directional environment using cellular automaton $[\mathrm{J}]$. Journal of Traffic and Transportation Engineering (English Edition), 2014, 1(6): 383392.

[8] Jin S, Qu X, Xu C, et al. An improved multi-value cellular automata model for heterogeneous bicycle traffic flow [J]. Physics Letters A, 2015, 379(39): 2409-2416.

[9] Ren G, Jiang H, Chen J, et al. Heterogeneous cellular automata model for straight-through bicycle traffic at signalized intersection [J]. Physica A: Statistical Mechanics and its Applications, 2016, 451: 70-83.

[10] Liu M, Xiong S. A refined and dynamic cellular automaton model for pedestrian-vehicle mixed traffic flow[J]. International Journal of Modern Physics C, 2016, 27(05):1650053.

[11] Li B, Xiong S, Duan P, et al. Traffic Safety Characteristics Analysis and Emergency Management Research on Campus[C]//Dependable Computing and Internet of Things (DCIT), 2015 2nd International Symposium on. IEEE, 2015: 106-111.

[12] Li B, Xiong S, Li X, et al. The Behavior Analysis of Pedestrian-cyclist Interaction at Non-signalized Intersection on Campus: Conflict and Interference[J]. Procedia Manufacturing, 2015, 3: 3345-3352. 